\title{
Implementation of an Output Feedback Controller in Operational Space
}

\author{
Qing Hua Xia*, Ser Yong Lim*, Marcelo H Ang Jr**, Tao Ming Lim* \\ *Singapore Institute of Manufacturing Technology \\ 71 Nanyang Drive \\ Singapore 638075 \\ **Mechanical Engineering Department \\ National University of Singapore \\ 10 Kent Ridge Crescent, Singapore 119260 \\ xqh66@yahoo.com, sylim@simtech.a-star.edu.sg,mpeangh@nus.edu.sg,tmlim@simtech.a-star.edu.sg
}

\begin{abstract}
This paper presents an operational space output feedback controller for non-redundant robot manipulators to achieve trajectory tracking without velocity measurements. The overall system can achieve a semi-global exponential stability (SGES) result for the position, orientation and velocity tracking errors as well as velocity observation errors. Experimental results of the proposed controller indicate good position and orientation tracking performance under parametric uncertainty and payload variations.
\end{abstract}

\section{INTRODUCTION}

Adaptive control of robot manipulators based on joint position and velocity measurements has been dealt with in great detail in the literature. In practice, many robotic systems are equipped with only high-precision link position measurement devices. Sensors to measure joint velocity are expensive and often contaminated by noise. Thus, a common practice is to approximate the velocity using backwards difference algorithm based on the joint position information, and use this estimation to design a controller. However, this approach cannot guarantee the closed-loop stability of the overall system. Moreover, it ignores the dynamic effect because of the position linearization across each sampling interval. Hence, it has limited applications in high precision control.

To overcome this drawback, some researchers have proposed advanced robot controllers that do not rely on link velocity measurements [1], [2], [3]. A detailed review on this topic can be found in [4]. In [5], a joint space controller based on an observed integrator backstepping technique was presented. This controller can achieve SGES result for the link position tracking error and the velocity observation error. The controller has then been further extended to the robot manipulator models that include actuator dynamics and joint flexibility [4], [6], [7].

However, in many robotic applications, tasks are defined in operational space [8]. Thus, it is more convenient to design a controller in the same space. Recently, a method for task space position tracking via quaternion feedback was presented in [9]. Pagilla et al. designed an adaptive output feedback controller which is shown to be semi-global asymptotically stable [10]. An observer-controller designed for task space tracking control using unit quaternion was proposed in [11].

Based on the controller structure presented in [5], we redesigned an output feedback controller in operational space. Simulation results indicated that, compared with the conventional computed-torque PD control using backwards difference approach to estimate velocities, the proposed controller can achieve higher position tracking accuracy under parametric uncertainty and payload variations [12]. This paper presents the implementation issues of the controller proposed in [12]. Experimental results verified the effectiveness and robustness of the proposed controller.

\section{ROBOT DYNAMIC MODEL}

In operational space [8], the end-effector equation of motion can be expressed as:

$$
A(x) \ddot{x}+B(x, \dot{x}) \dot{x}+G(x)=F
$$

where $F$ is the $n \times 1$ operational force vector, $x$ is the $n \times 1$ vector describing the position and orientation of the end-effector, $A(x)$ is the $n \times n$ kinetic energy matrix, $B(x, \dot{x})$

is the $n \times n$ centrifugal and Coriolis matrix, and $G(x)$ is the $n \times 1$ vector of gravitational forces.

The end-effector velocity is related to joint velocity by:

$$
\dot{x}=J(q) \dot{q}
$$

where $J(q)$ is the basic Jacobian of a robot, $q$ represents 
joint position vector, and $\dot{q}$ represents joint velocity vector.

Joint torque and operational force vector is related by:

$$
\Gamma=J^{T} F
$$

\section{PROPERTIES OF A ROBOT DYNAMIC MODEL}

Property I: The $n \times n$ kinetic energy matrix $A(x)$ defined in (1) satisfies the following inequality [14]:

$$
m_{1}\|z\|^{2} \leq z^{T} A(x) z \leq m_{2}\|z\|^{2} \quad \forall z \in \mathfrak{R}^{n}
$$

where $m_{1}$ and $m_{2}$ are known positive scalar constants. \|.. represents the standard Euclidean norm.

Property II: For non-redundant robots, $B(x, \dot{x})$ in (1) satisfies the following relationships [12]:

$$
\begin{gathered}
z^{T}\left[\frac{1}{2} \dot{A}(x)-B(x, \dot{x})\right] z=0 \quad \forall z \in \mathfrak{R}^{n} \\
B(x, y) z=B(x, z) y \quad \forall y, z \in \mathfrak{R}^{n}
\end{gathered}
$$

and

$$
\|B(x, \dot{x})\|_{i \infty} \leq \zeta_{c}\|\dot{x}\|
$$

where $\zeta_{c}$ is a known positive scalar constant and \|\|$_{i \infty}$ represents the matrix induced infinity norm [15].

\section{OUTPUT FEEDBACK CONTROLLER}

The control objective is to develop an end-effector position and orientation tracking controller in operational space for the robot described by (1) with position information only.

First, define the $n \times 1$ end-effector position and orientation tracking error as:

$$
e=x_{d}-x
$$

where $x_{d}$ represents the desired end-effector position and orientation trajectory. The requirement for the trajectory is that $x_{d}$ and its first and second derivatives are all bounded function of time. We can place the following bound for $x_{d}$ :

$$
\left\|\dot{x}_{d}\right\| \leq \zeta_{d}
$$

where $\zeta_{d}$ is a known positive scalar constant.

\section{A. Velocity Observer Formulation}

To estimate the end-effector velocity measurements, we use the following second order velocity observer:

$$
\begin{gathered}
\dot{\hat{x}}=y+k \tilde{x} \\
\dot{y}=A^{-1}(x)[F-B(x, \dot{\hat{x}}) \dot{\hat{x}}-G(x)]
\end{gathered}
$$

where

$$
\tilde{x}=x-\hat{x}
$$

$y$ is an $n \times 1$ auxiliary variable, and $k$ is a positive scalar constant defined by:

$$
k=\frac{1}{m_{1}}\left(\zeta_{c} \zeta_{d}+\zeta_{c} k_{0}+\zeta_{c} k_{s} k_{0}+k_{s}+2 k_{n}\right)
$$

$F$ in (11) is the control input to the observer, it is generated by the controller indicated by (15). $k_{0}, k_{s}$ and $k_{n}$ are positive scalar control gains, $\zeta_{c}$ and $\zeta_{d}$ are declared in (7) and (9) respectively. Differentiating (12) with respect to time, we can form the following velocity observation error:

$$
\dot{\tilde{x}}=\dot{x}-\dot{\hat{x}}
$$

\section{B. Controller Formulation}

Based on the structure of the above observer, we use the following controller to generate the required force:

$$
F=\left(k_{s}+k_{n d}\right) \eta_{p}+w_{e}
$$

where $k_{n d}$ is a positive controller gain defined as:

$$
k_{n d}=2 k_{n}+\zeta_{c} k_{0}+\left(k_{s} m_{2}+k m_{2}\right)^{2} k_{n}
$$

and the $n \times 1$ auxiliary terms, $\eta_{p}$ and $w_{e}$ are defined as:

$$
\begin{gathered}
\eta_{p}=\dot{x}_{d}+k_{s} e-\dot{\hat{x}} \\
w_{e}=A(x)\left[\ddot{x}_{d}+k_{s}\left(\dot{x}_{d}-\dot{\hat{x}}\right)\right]+B(x, \dot{\hat{x}})\left(\dot{x}_{d}+k_{s} e\right)+G(x)
\end{gathered}
$$


The force $F$ command will be used for the observer indicated by (10) and (11). And the torque command for driving the robot can be obtained by (3).

\section{STABILITY RESULT}

For the observer and controller presented in the previous section, if the exact model of a robot is known, then the position and orientation tracking error defined in (8) is SGES according to the following theorem [12].

Theorem: Provided the output feedback controller gains satisfy the following sufficient conditions:

$$
\begin{array}{r}
k_{s}>\frac{1}{k_{n}} \\
k_{0}>\sqrt{\frac{\lambda_{2}}{\lambda_{1}}}\|\operatorname{err}(0)\|
\end{array}
$$

the closed-loop observation tracking error system is SGES as illustrated by:

$$
\|\operatorname{err}(t)\| \leq \sqrt{\frac{\lambda_{2}}{\lambda_{1}}}\|\operatorname{err}(0)\| e^{-\lambda t}
$$

where

$$
\begin{gathered}
\lambda_{1}=\min \left\{m_{1}, 1\right\}, \lambda_{2}=\max \left\{m_{2}, 1\right\}, \lambda=\frac{\lambda_{3}}{\lambda_{2}}, \lambda_{3}=k_{s}-\frac{1}{k_{n}} \\
e r r=\left[\begin{array}{ccc}
e^{T} & \eta_{p}^{T} & \dot{\widetilde{x}}^{T}
\end{array}\right]^{T} \in \mathfrak{R}^{3 n}
\end{gathered}
$$

The overall system stability analysis can be found in [12].

\section{FORMULATION OF ESTIMATION ERROR}

To facilitate the estimation error formulation, we rewrite (10) as following:

$$
\dot{\hat{x}}=y+k(x-\hat{x})
$$

To formulate the position and orientation estimation error in close form, we rewrite (23) as following:

$$
J(q) \dot{\hat{q}}=y+k[x(q)-\hat{x}(\hat{q})]
$$

where $\hat{q}$ is the $n \times 1$ vector of the estimated joint positions, $\dot{\hat{q}}$ is the $n \times 1$ vector of the estimated joint velocities.
We assume that a robot always works in a non-singular region so that the inverse of $J(q)$ is possible, and for a non-redundant robot, the mapping from $\dot{\hat{x}}$ to $\dot{\hat{q}}$ is unique. Rewrite (24) as:

$$
\dot{\hat{q}}=J^{-1}(q)\{y+k[x(q)-\hat{x}(\hat{q})]\}
$$

From (25), we can get the estimated joint positions $\hat{q}$, and $\hat{x}$ can be expressed explicitly as a function of $\hat{q}$.

Since the actual joint positions information are available, the actual position and orientation of the end-effector can be obtained by evaluating the homogeneous transformation matrix $T(q)$ of a robot:

$$
T(q)=\left[\begin{array}{cccc}
n_{x} & o_{x} & a_{x} & p_{x} \\
n_{y} & o_{y} & a_{y} & p_{y} \\
n_{z} & o_{z} & a_{z} & p_{z} \\
0 & 0 & 0 & 1
\end{array}\right]
$$

Similarly, from $\hat{q}$, the estimated position and orientation of the end-effector can be written as:

$$
T(\hat{q})=\left[\begin{array}{cccc}
\hat{n}_{x} & \hat{o}_{x} & \hat{a}_{x} & \hat{p}_{x} \\
\hat{n}_{y} & \hat{o}_{y} & \hat{a}_{y} & \hat{p}_{y} \\
\hat{n}_{z} & \hat{o}_{z} & \hat{a}_{z} & \hat{p}_{z} \\
0 & 0 & 0 & 1
\end{array}\right]
$$

The position estimation error can be easily expressed as:

$$
\delta p=\left[\begin{array}{lll}
p_{x}-\hat{p}_{x} & p_{y}-\hat{p}_{y} & p_{z}-\hat{p}_{z}
\end{array}\right]^{T}
$$

The orientation estimation error can be expressed as [13]:

$$
\begin{aligned}
\delta \phi=- & \frac{1}{2}\left(\left[\begin{array}{ccc}
0 & -n_{z} & n_{y} \\
n_{z} & 0 & -n_{x} \\
-n_{y} & n_{x} & 0
\end{array}\right]\left[\begin{array}{l}
\hat{n}_{x} \\
\hat{n}_{y} \\
\hat{n}_{z}
\end{array}\right]\right. \\
& +\left[\begin{array}{ccc}
0 & -o_{z} & o_{y} \\
o_{z} & 0 & -o_{x} \\
-o_{y} & o_{x} & 0
\end{array}\right]\left[\begin{array}{l}
\hat{o}_{x} \\
\hat{o}_{y} \\
\hat{o}_{z}
\end{array}\right] \\
& \left.+\left[\begin{array}{ccc}
0 & -a_{z} & a_{y} \\
a_{z} & 0 & -a_{x} \\
-a_{y} & a_{x} & 0
\end{array}\right]\left[\begin{array}{l}
\hat{a}_{x} \\
\hat{a}_{y} \\
\hat{a}_{z}
\end{array}\right]\right)
\end{aligned}
$$


And the close form of the position and orientation observation error can be written as:

$$
\tilde{x}=\left[\begin{array}{ll}
\delta p^{T} & \delta \phi^{T}
\end{array}\right]^{T}
$$

\section{EXPERIMENTAL RESULTS}

The experiment was performed using PUMA 560 robot as shown in Fig. 1.

We bypassed the original motion controller card of PUMA 560 and use Servo To Go controller card to control the robot. Our real-time robotic control software runs under Windows NT 4.0 with RTX 4.3.2.1. The PC used is a dual-processor $800 \mathrm{MHz} \mathrm{PC}$, and the sampling time is selected to be $1 \mathrm{~ms}$.

Our task is to move the end-effector with the desired position trajectory indicated by (31) while maintaining the initial end-effector orientation all the time.

$$
\begin{aligned}
& P x_{d}=P x_{0}+50 \sin (2 \pi \mathrm{ft})\left(1-\mathrm{e}^{-0.05 \mathrm{t}^{3}}\right) \mathrm{mm}, \\
& P y_{d}=P y_{0}+50 \cos (2 \pi \mathrm{ft})\left(1-\mathrm{e}^{-0.05 \mathrm{t}^{3}}\right) \mathrm{mm}, \\
& P z_{d}=P z_{0}+50 \cos (2 \pi \mathrm{ft})\left(1-\mathrm{e}^{-0.05 \mathrm{t}^{3}}\right) \mathrm{mm}, \\
& f=0.1 \mathrm{~Hz}
\end{aligned}
$$

where $P x_{0}, P y_{0}$ and $P z_{0}$ are the initial positions of the robot.

The exponential terms are to ensure that the initial desired velocities and accelerations are all zeros.

In our experiments, we used the combination of (25) and (11) to form our velocity observer, and use (28), (29) and (30) to form the position and orientation estimation error.

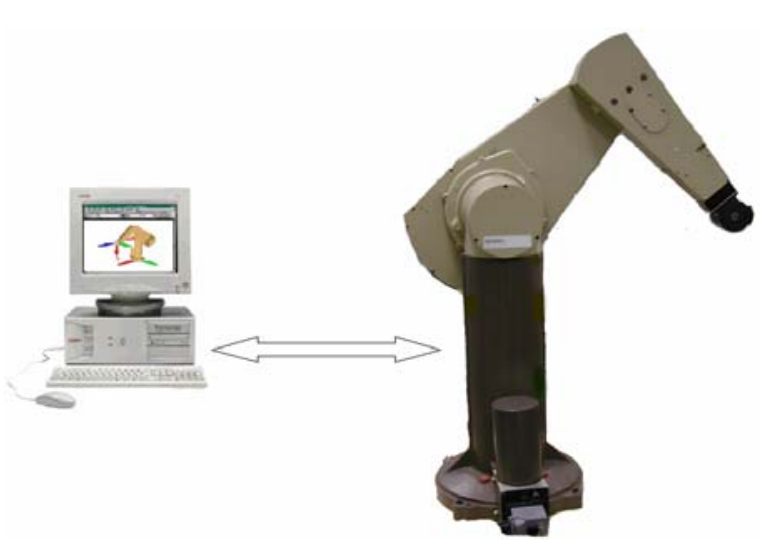

Fig. 1 Experimental setup of PUMA 560

\section{A. Tracking Error Formulation}

In section 4.2, the proposed controller needs to use $x_{d}, \dot{x}_{d}$ and $\ddot{x}_{d}$ to generate the required force commands. For position control, we can get the desired end-effector position, linear velocities and accelerations from (31). The position tracking errors $e_{p o s}$ can be easily obtained by:

$$
e_{p o s}=\left[\begin{array}{lll}
P x_{d}-p_{x} & P y_{d}-p_{y} & P z_{d}-p_{z}
\end{array}\right]^{T}
$$

For orientation control, since the task is to maintain the initial end-effector orientation during position tracking, the desired end-effector angular velocities and accelerations are just zeros. And the orientation tracking errors $e_{\text {ori }}$ can be expressed as [13]:

$$
\begin{aligned}
e_{\text {ori }}= & \frac{1}{2}\left(\left[\begin{array}{ccc}
0 & -n_{z} & n_{y} \\
n_{z} & 0 & -n_{x} \\
-n_{y} & n_{x} & 0
\end{array}\right]\left[\begin{array}{l}
n_{x i n i} \\
n_{y i n i} \\
n_{z i n i}
\end{array}\right]\right. \\
& +\left[\begin{array}{ccc}
0 & -o_{z} & o_{y} \\
o_{z} & 0 & -o_{x} \\
-o_{y} & o_{x} & 0
\end{array}\right]\left[\begin{array}{c}
o_{x i n i} \\
o_{y i n i} \\
o_{z i n i}
\end{array}\right] \\
& \left.+\left[\begin{array}{ccc}
0 & -a_{z} & a_{y} \\
a_{z} & 0 & -a_{x} \\
-a_{y} & a_{x} & 0
\end{array}\right]\left[\begin{array}{l}
a_{x i n i} \\
a_{y i n i} \\
a_{z i n i}
\end{array}\right]\right)
\end{aligned}
$$

where the initial end-effector orientation can be obtained from the robot homogeneous transformation matrix $T_{i n i}(q)$ :

$$
T_{i n i}(q)=\left[\begin{array}{cccc}
n_{x i n i} & o_{x i n i} & a_{x i n i} & p_{x i n i} \\
n_{y i n i} & o_{y i n i} & a_{y i n i} & p_{y i n i} \\
n_{z i n i} & o_{z i n i} & a_{z i n i} & p_{z i n i} \\
0 & 0 & 0 & 1
\end{array}\right]
$$

\section{B. Experimental Results with Parametric Uncertainty}

In order to adjust the commanded force in different directions separately, we selected different values for the diagonal terms of the diagonal gains matrices.

To test what are the smallest tracking errors that can be achieved by the proposed controller, we increased the controller gains until the robot started to vibrate, the gains 
used are listed in (35):

$$
\begin{aligned}
& k_{n d}=\operatorname{diag}\{220,220,220,66,66,66\} \\
& k=\operatorname{diag}\{200,200,200,60,60,60\} \\
& k_{s}=\operatorname{diag}\{180,180,180,54,54,54\}
\end{aligned}
$$

In order to make comparison, we also implemented the controller indicated by (15), (16), (17) and (18) but the estimated velocities in (18) were replaced by that obtained from backwards difference plus a low pass filter with cutoff frequency of $100 \mathrm{~Hz}$, and we called it the backwards difference controller. During the experiments, we found that the controller gains values $k_{s}$ and $k_{n d}$ indicated in (35) were too high for the backwards difference controller, they caused the system to vibrate. We then selected the gains listed in (36) in order to reduce vibration:

$$
\begin{aligned}
& k_{n d}=\operatorname{diag}\{20,20,20,12,12,12\} \\
& k_{s}=\operatorname{diag}\{90,90,90,30,30,30\}
\end{aligned}
$$

The experimental results are shown in Table I and Fig. 2. Where OFB stands for the output feedback controller, and $\mathrm{BD}$ for the backwards difference controller.

The results show that, using the proposed output feedback controller, the maximum position and orientation tracking errors are about 1.4 to 3.5 times smaller than that of the backwards difference controller.

TABLE I

MAXIMUM POSITION AND ORIENTATION TRACKING ERRORS

\begin{tabular}{|l|c|c|c|c|c|c|}
\hline & $e_{x}$ & $e_{y}$ & $e_{z}$ & $\delta_{x}$ & $\delta_{y}$ & $\delta_{z}$ \\
\hline OFB & $0.7 \mathrm{~mm}$ & $0.5 \mathrm{~mm}$ & $0.5 \mathrm{~mm}$ & $0.08^{\circ}$ & $0.04^{\circ}$ & $0.09^{\circ}$ \\
\hline BD & $1.0 \mathrm{~mm}$ & $0.7 \mathrm{~mm}$ & $1.5 \mathrm{~mm}$ & $0.20^{\circ}$ & $0.14^{\circ}$ & $0.22^{\circ}$ \\
\hline
\end{tabular}

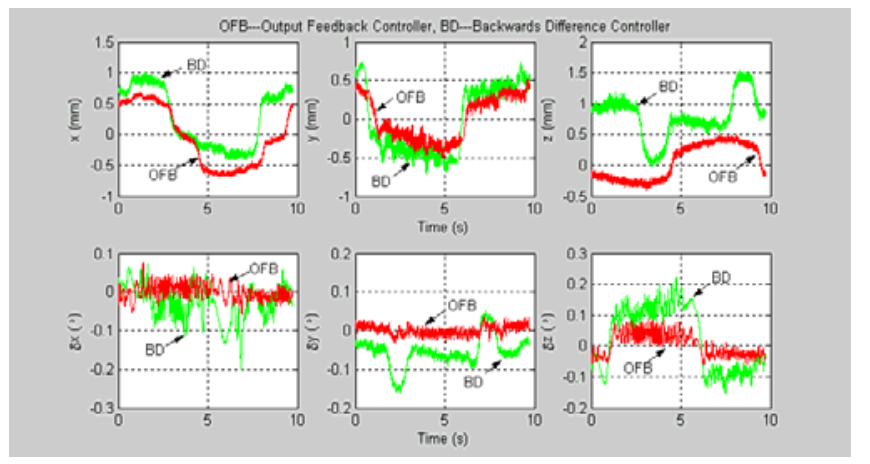

Fig. 2 Tracking errors with parametric uncertainty

\section{Experimental Results with Payload Variations}

To examine the performance of the proposed output feedback controller and the backwards difference controller under payload variations, a payload of $1.5 \mathrm{~kg}$ was attached to the end-effector of PUMA 560. Using the same gains as listed in (35) and (36) for the output feedback controller and the backwards difference controller, respectively, the experimental results are shown in Table II.

The results show that, using the output feedback controller, the maximum position and orientation tracking errors are about 2.4 to 9.2 times smaller than that of the backwards difference controller.

Remark I: The experimental results indicate that, compared with the backwards difference controller, the output feedback controller seems less sensitive to payload variations, which indicates that the proposed output feedback controller maybe robust against parametric uncertainty and payload variations. The limitation of the backwards difference controller in compensation of parametric uncertainty and payload variations is because of its linear behavior. While the output feedback controller is designed to mimic the dynamic behavior of a robot, the position and orientation tracking performance can be better if the robot dynamic model is accurate enough.

Remark II: Note that the gains $k$ in (13) and $k_{n d}$ in (16) are selected directly rather than calculated online.

Remark III: The proposed output feedback controller seems complex, but it is easy to see from (10), (11) and (15) that this controller only requires the selection of three controllers gains $k_{n d}, k$ and $k_{s}$. After examining (13) and (16), it is easy to see that $k_{n d}>k>k_{s}$. But in our experiments, even if the gains selection does not meet the above mentioned constrain, the robot still works well. We mention it here to indicate that, owing to the conservative nature of the Lyapunov stability analysis, the gains condition is treated as selection guideline rather than an absolute mandate.

TABLE II

MAXIMUM POSITION AND ORIENTATION TRACKING ERRORS

\begin{tabular}{|l|c|c|c|c|c|c|}
\hline & $e_{x}$ & $e_{y}$ & $e_{z}$ & $\delta_{x}$ & $\delta_{y}$ & $\delta_{z}$ \\
\hline OFB & $0.8 \mathrm{~mm}$ & $0.5 \mathrm{~mm}$ & $0.7 \mathrm{~mm}$ & $0.07^{\circ}$ & $0.04^{\circ}$ & $0.10^{\circ}$ \\
\hline BD & $1.9 \mathrm{~mm}$ & $1.4 \mathrm{~mm}$ & $3.1 \mathrm{~mm}$ & $0.30^{\circ}$ & $0.37^{\circ}$ & $0.33^{\circ}$ \\
\hline
\end{tabular}


Remark $I V$ : The gain condition given by (19) implies that $k_{0}$ can be selected to cover any set of initial condition of the error vector $\operatorname{err}(t)$. In addition, from (20) and (21) we can see that, the exponential transient response of end-effector position and orientation tracking errors can be sped up by increasing the controller gains $k_{s}$ and $k_{n}$.

Remark $V$ : To get the estimated joint velocity $\dot{\hat{q}}$ from (25),

the inverse of $J(q)$ is required, and we need to control robot motion to avoid the singular region of a robot.

\section{CONCLUSIONS}

In this paper, we present the implementation issues of an operational space output feedback controller for non-redundant manipulators. Compared with the same design technique in joint space, operational space controller design is more practical. And experimental results verify the robustness and good position and orientation tracking performance of the nonlinear controller under parametric uncertainty and payload variations.

\section{REFERENCES}

[1] H. Berghuis and H. Nijmeijer, "Robust control of robots using only position measurements," Proc. IFAC World Congress, Sydney, Australia, vol. 1, pp. 501-506, July 1993.

[2] H. Berghuis and H. Nijmeijer, "A passivity approach to controller-observer design for robots," IEEE Trans. Robot. Automat., vol. 9, pp. 740-754, Dec. 1993.

[3] S. Nicosia and P. Tomei, "Robot control by using only joint position measurements," IEEE Trans. Automat. Contr., vol. 35, pp. 1058-1061, Sept. 1990.

[4] S. Y. Lim, D. Dawson, and M. Queiroz, "A partial state feedback controller for trajectory tracking of rigid-link flexible-joint robots using an observed backstepping approach," Proc. Amer. Contr. Conf., Seattle, WA, pp. 4300-4304, June 1994.
[5] S. Y. Lim, D. Dawson, and K. Anderson, "Reexamining Nicosia's robot observer-controller from abackstepping perspective," IEEE Trans. on Control System Technology, 4(3), pp. 304-310. May 1996.

[6] S. Y. Lim, J. Hu, and D. Dawson, "An output feedback controller for trajectory tracking of RLED robots using an observed backstepping approach," Proc. IEEE Conf. Contr. Applicat. Glasgow,Scotland, pp. 71-76, Aug, 1994.

[7] S. Y. Lim, D. Dawson, J. Hu, and M. Queiroz, "An adaptive link position tracking controller for rigid-link flexible-joint robots without velocity measurements," Proc. IEEE Conf. Decis. Control, Lake Buena Vista, FL, pp. 351-356, Dec. 1994.

[8] O. Khatib, "A unified approach to motion and force control of robot manipulators: the operational space formulation," IEEE J. on Robotics and Automation, vol. 3, no. 1, 1987.

[9] B. Xian, M. Queiroz, D. Dawson, I. Walker, "Task-space tracking control of redundant robot manipulators via quaternion feedback," Proc. Of the 2001 IEEE Conf. on Control Applications, pp. 363-368, 2001.

[10] P. R. Pagilla and M. Tomizuka, "An Adaptive Output Feedback Controller for Robot Arms: Stability and Experiments," Automatica, Journal of the International Federation of Automatic Control, vol. 37, no. 7, pp. 983-995, July 2001.

[11]F. Caccavale, C. Natale, and L. Villani, "Task-space control without velocity measurements," Proc. Of the International Conf. on Robotics and Automation, Detroit, MI, pp. 512-517, 1999.

[12] Q. H. Xia, S. Y. Lim and M. H. Ang Jr., "An operational space observer-controller for trajectory tracking," The $11^{\text {th }}$ International Conference on Advanced Robotics, University of Coimbra, Portugal, June 30-July 3, 2003.

[13] O. Khatib, Advanced Robotics: Robots \& the Human, Professional Activities Centre, Faculty of Engineeing, National University of Singapore, 2002.

[14] M. Spong and M. Vidyasagar, Robot Dynamics and Control. New York: Wiley, 1989.

[15]M. Vidyasagar, Nonlinear Systems Analysis. Englewood Cliffs, NJ: Prentice Hall, 1978. 